\title{
Nod Factors of Azorhizobium caulinodans Strain ORS571 Can Be Glycosylated with an Arabinosyl Group, a Fucosyl Group, or Both
}

\author{
Peter Mergaert, ${ }^{1}$ Myriam Ferro, ${ }^{2}$ Wim D'Haeze, ${ }^{1}$ Marc Van Montagu, ${ }^{1}$ Marcelle Holsters, ${ }^{1}$ \\ and Jean-Claude Promé ${ }^{2}$ \\ ${ }^{1}$ Laboratorium voor Genetica, Department of Genetics, Flanders Interuniversity Institute for Biotechnology \\ (VIB), Universiteit Gent, K.L. Ledeganckstraat 35, B-9000 Gent, Belgium; ' Laboratoire de Pharmacologie \\ et Toxicologie Fondamentales, Spectrometrie de Masse et Signaux Biologiques, Centre National de la \\ Recherche Scientifique, 205, route de Narbonne, F-31077 Toulouse Cedex, France \\ Received 16 December 1996. Accepted 20 March 1997.
}

In addition to the previously described arabinosylated Nod factors, Azorhizobium caulinodans can also produce fucosylated Nod factors and Nod factors that are both arabinosylated and fucosylated. The presence of a plasmid carrying extra copies of a subset of nod genes as well as bacterial growth conditions influence the relative proportion of carbamoylated, fucosylated, and arabinosylated Nod factors. By using a root hair formation assay, we demonstrate that the Nod factor glycosylations are important for biological activity on Sesbania rostrata roots.

To trigger the nodulation process of host plants, rhizobia produce Nod factors. Nod factors form a class of lipo-chitooligosaccharide (LCO) molecules constituted of a chitooligosaccharide $(\beta-1,4-$ linked $N$-acetylglucosamine [GlcNAc]) to which a fatty acid is attached at the nonreducing end, replacing the $N$-acetyl group. The fine structure of Nod factors depends on the Rhizobium strain. Nod factors differ in the oligosaccharide chain length, the nature of the fatty acid, and the substitutions on the reducing and nonreducing ends of the oligosaccharide (Dénarié et al. 1996). Specific Nod factor structures are needed for activity on the host plants, and therefore Nod factors are major contributors to the host range determination.

We previously described structures of Nod factors produced by Azorhizobium caulinodans strain ORS571 (NodARc), a symbiont of the tropical legume Sesbania rostrata (Mergaert et al. 1993). In that study, an overproducing strain, ORS571(pRG70), was used. The plasmid pRG70 carries the nod genes of ORS571 needed for Nod factor synthesis, except nodD and nolK (Mergaert et al. 1993). NodARc produced by ORS571(pRG70) were mainly pentameric with a minor fraction of

Corresponding author: M. Van Montagu; Fax: 32-9-2645349;

E-mail: mamon@gengenp.rug.ac.be

Present address of P. Mergaert: Institut des Sciences Végétales, Centre National de la Recherche Scientifique, Avenue de la Terrasse, F-91198, Gif-sur-Yvette, Cedex, France. tetrameric forms. The acyl chain was either vaccenic (C18:1) or stearic (C18:0) acid. At the nonreducing end of the oligosaccharide, an $N$-methyl group was present in all molecules, and a $6-O$-carbamoyl group was present on about $60 \%$ of the LCOs. The reducing end was branched with an arabinosyl group in approximately $30 \%$ of the population.

Here, we show that bacterial culture conditions and the presence of extra copies of a subset of nod genes influence Nod factor structure in several ways.

Cultures of ORS571 and ORS571(pRG70) were grown in 10-liter flasks containing 10 liters of MMO medium (Mergaert et al. 1993). Precultures $(100 \mathrm{ml})$ were grown overnight at $37^{\circ} \mathrm{C}$ and added to the 10 liters of fresh medium. The cultures were incubated at $37^{\circ} \mathrm{C}$ and aerated by pumping air and agitating them with a magnetic stirrer. One hour after dilution, naringenin was added to a final concentration of $20 \mu \mathrm{M}$ to induce Nod factor synthesis. The cultures were grown for $16 \mathrm{~h}$ after induction to allow accumulation of Nod factors. These growth conditions were different from the ones used previously because the cultures were more strongly aerated. Nod factors were extracted with $n$-butanol and purified with $\mathrm{C} 18$ reverse-phase high-pressure liquid chromatography (RPHPLC) as described before (Mergaert et al. 1993), except that cells were not removed because we found that large amounts of Nod factors remained associated with the bacteria (Fernández-López et al. 1996). RP-HPLC resulted in two peaks, PI and PII, containing Nod factors. PI and PII from ORS571 and ORS571(pRG70) were analyzed with fast-atom bombardment mass spectrometry (FAB-MS). The spectra were recorded as before (Mergaert et al. 1996). FAB-MS spectra of PII preparations from ORS571(pRG70) and ORS571 are shown in Figure 1A and B, respectively. PI gave the same peaks, except that they were 2 mass units lower because different fatty acids (C18:1 for PI and C18:0 for PII) were present (Mergaert et al. 1993).

FAB-MS revealed several notable differences in the Nod factor populations of the previous and the present studies. In the PII fraction of the previous study (Mergaert et al. 1993), $[\mathrm{M}+\mathrm{H}]^{+}$ions were observed at $\mathrm{m} / z, 1447$ and 1315 correspond- 
ing to pentameric NodARc, carrying a methyl, carbamoyl, and arabinosyl modification and a methyl and carbamoyl modification, respectively. Noncarbamoylated species at 43 mass units below were also present. The current PII fractions from both ORS571 and ORS571(pRG70) did not exhibit prominent $[\mathrm{M}+\mathrm{H}]^{+}$ions corresponding to noncarbamoylated species but showed a nearly complete carbamoylation of Nod factors. They showed additional $[\mathrm{M}+\mathrm{H}]^{+}$ions at $\mathrm{m} / z, 1461$ and 1593 . Their relative abundance was higher in the PII fraction of ORS571 than in that of ORS571(pRG70) (Fig. 1A and B).
The mass difference between these new species and the previous ones is $146 \mathrm{Da}$, corresponding to a deoxyhexose identified as fucose by determination of the carbohydrate composition (see below). A final difference between the current and previous NodARc preparations was the presence in PI of a small amount of LCOs carrying a palmitoyl (C16:0) chain (see below).

Differences between the present results and the previous ones concerning the overproducing strain ORS571(pRG70) most probably were caused by different physiological condi-
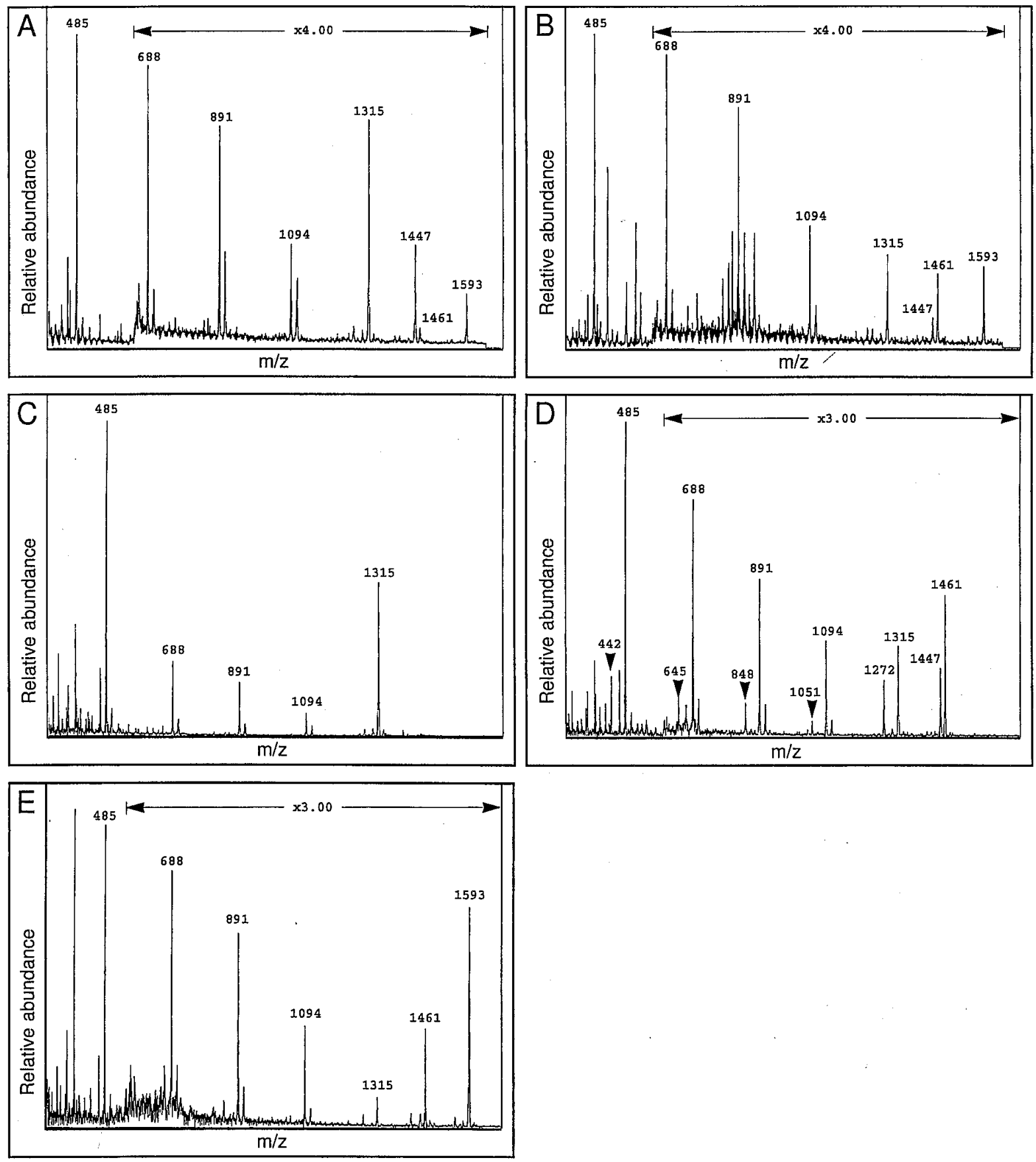

Fig 1. Fast-atom bombardment mass spectra. A, PII of ORS571(pRG70); B, PII of ORS571; C, PIId of ORS571; D, PIIe of ORS571; and E, PIIf of ORS571. 
tions of bacteria grown in a more or less aerated environment. They were not caused by modifications in the extraction procedure (supernatant versus whole-culture extractions) because neither qualitative nor quantitative differences were detected when the culture medium or the bacterial cells were extracted (data not shown). The relative enhancement of the production of fucosylated LCOs by the ORS571 wild-type strain can be explained by the fact that the nolK gene was not amplified in ORS571(pRG70), resulting in an imbalance of Nod proteins and thereby decreasing fucosylation. Indeed, we have previously suggested that NolK may be involved in the synthesis of GDP-fucose, the fucosyl donor in fucosyltransferase reactions (Mergaert et al. 1996).

To separate the different compounds in the RP-HPLC peaks PI and PII, an additional HPLC step, in which a polyol
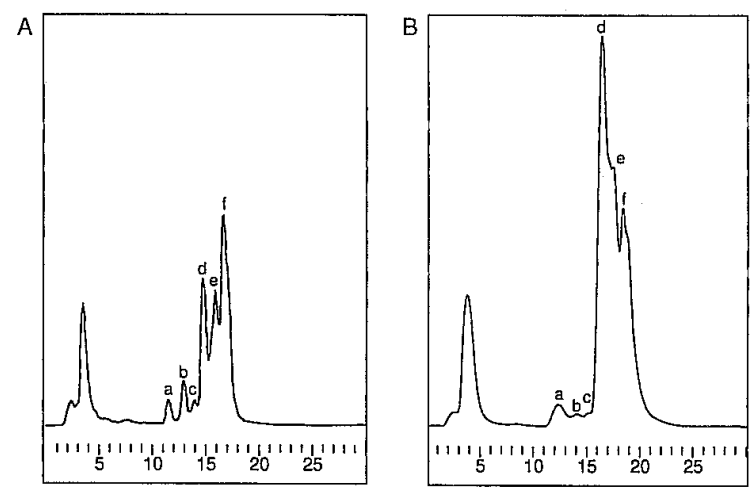

Fig. 2. Polyol-HPLC (high-pressure liquid chromatography) profiles of ORS571 Nod factors. Reverse-phase HPLC fractions PI from ORS571 (A) and ORS571(pRG70) (B) were purified on a polyol HPLC column. Peaks a to f contain Nod factors. The profiles for PII were very similar (data not shown). column $(4.6 \times 250 \mathrm{~mm}$, carbohydrate $\mathrm{OH}-100,7 \mu$; Alltech, Deerfield, IL) was used, was included in the purification protocol. A gradient from 87.5 to $62.5 \%$ acetonitrile in $30 \mathrm{~min}$ was applied. Detection of LCOs was at $206 \mathrm{~nm}$. Under these conditions, LCOs eluted between 11 and $18 \mathrm{~min}$. The profiles of the chromatograms of PI and PII displayed three minor (a, $\mathrm{b}$, and c) and three major (d, e, and f) LCO-containing peaks (Fig. 2). The dominant product for ORS571 was peak f (Fig. 2A) and for ORS571(pRG70) peak d (Fig. 2B). The different fractions were analyzed by FAB-MS. The results are listed in Table 1, and the structure of the fractions is given in Figure 3. Figure 1C, D, and E show the spectra of the ORS571 fractions PIId, PIIe, and PIIf, respectively. Series of peaks separated by 203 mass units corresponded to fragmentations of the chitooligosaccharide chain of LCOs, and these sequences were confirmed by MS-MS spectra of the $[\mathrm{M}+\mathrm{H}]^{+}$ions. Thus, in Figure $1 \mathrm{C}$, the $[\mathrm{M}+\mathrm{H}]^{+}$ion is at $m / z, 1315$. The fragment at $\mathrm{m} / z$ 1094 corresponded to the loss of GlcNAc-OH, which indicates the absence of a substitution at the reducing end. Other fragments formed a series separated by 203 mass units ending at $m / z 485$, corresponding to the nonreducing end of the oligosaccharide, which is both $N$-methylated and $N$-acylated by stearic acid and $O$-carbamoylated. The spectrum of fraction

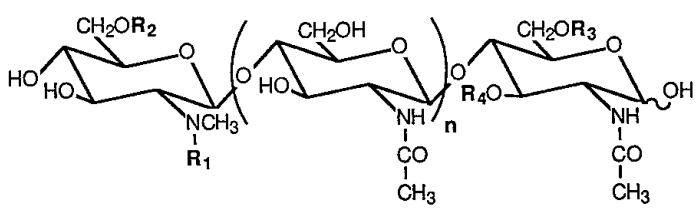

Fig. 3. Structure of Nod factors from Azorhizobium caulinodans. For the identity of the side groups R1-R4 and oligosaccharide chain length $(n)$, see Table 1.

Table 1. Summary of lipo-chitooligosaccharides produced by Azorhizobium caulinodans

\begin{tabular}{|c|c|c|c|c|c|c|}
\hline Fraction $^{a}$ & {$[\mathbf{M}+\mathbf{H}]^{+\mathbf{b}}$} & $\mathbf{R}_{1}{ }^{\mathbf{c}}$ & $\mathbf{R}_{\mathbf{2}}$ & $\mathbf{R}_{\mathbf{3}}$ & $\mathbf{R}_{\mathbf{4}}$ & $n$ \\
\hline \multirow[t]{2}{*}{ PIa } & 1084 & $\mathrm{C} 16: 0$ & Carbamoyl & $\mathrm{H}$ & $\mathrm{H}$ & 2 \\
\hline & 1110 & C18:1 & Carbamoyl & $\mathrm{H}$ & $\mathrm{H}$ & 2 \\
\hline \multirow[t]{2}{*}{ PIb } & 1230 & C16:0 & Carbamoyl & Fucosyl & $\mathrm{H}$ & 2 \\
\hline & 1256 & C18:1 & Carbamoyl & Fucosyl & $\mathrm{H}$ & 2 \\
\hline PIc & 1388 & C18:1 & Carbamoyl & Fucosyl & Arabinosyl & 2 \\
\hline \multirow[t]{2}{*}{ PId } & 1287 & C16:0 & Carbamoyl & $\mathrm{H}$ & $\mathrm{H}$ & 3 \\
\hline & 1313 & C18:1 & Carbamoyl & $\mathrm{H}$ & $\mathrm{H}$ & 3 \\
\hline \multirow[t]{4}{*}{ PIe } & 1244 & C16:0 & $\mathrm{H}$ & $\mathrm{H}$ & $\mathrm{H}$ & 3 \\
\hline & 1270 & C18:1 & $\mathrm{H}$ & $\mathrm{H}$ & $\mathrm{H}$ & 3 \\
\hline & $1445^{\mathrm{d}}$ & C18:1 & Carbamoyl & $\mathrm{H}$ & Arabinosyl $^{\mathrm{e}}$ & 3 \\
\hline & $1459^{d}$ & C18:1 & Carbamoyl & Fucosyl & $\mathrm{H}$ & 3 \\
\hline PIf & 1591 & C18:1 & Carbamoyl & Fucosyl & Arabinosyl & 3 \\
\hline PIIa & 1112 & C18:0 & Carbamoyl & $\mathrm{H}$ & $\mathrm{H}$ & 2 \\
\hline PIIb & 1258 & C18:0 & Carbamoyl & Fucosyl & $\mathrm{H}$ & 2 \\
\hline PIIc & 1390 & C18:0 & Carbamoyl & Fucosyl & Arabinosyl & 2 \\
\hline PIId & 1315 & C18:0 & Carbamoyl & $\mathrm{H}$ & $\mathrm{H}$ & 3 \\
\hline \multirow{3}{*}{ PIIe } & 1272 & C18:0 & $\mathrm{H}$ & $\mathrm{H}$ & $\mathrm{H}$ & 3 \\
\hline & $1447^{d}$ & C18:0 & Carbamoyl & $\mathrm{H}$ & Arabinosyle & 3 \\
\hline & $1461^{\mathrm{d}}$ & C18:0 & Carbamoyl & Fucosyl & $\mathrm{H}$ & 3 \\
\hline PIIf & 1593 & C18:0 & Carbamoyl & Fucosyl & Arabinosyl & 3 \\
\hline
\end{tabular}

\footnotetext{
${ }^{a}$ Polyol high-pressure liquid chromatography fraction.

${ }^{\mathrm{b}}$ Determined by fast-atom bombardment mass spectrometry (FAB-MS) and MS-MS.

${ }^{c}$ C16:0, palmitoyl; C18:1, vaccenoyl; and C18:0, stearoyl.

${ }^{\mathrm{d}}$ For ORS571(pRG70), the arabinosylated compound is most abundant and the fucosylated compound is nearly absent in this fraction, whereas for ORS571, the fucosylated compound is most abundant.

e The arabinosyl group can also be located on O-6 $\left(\mathrm{R}_{3}\right)$.
} 
PIIe (Fig. 1D) indicated a mixture of compounds. The series of ions separated by 203 mass units from $\mathrm{m} / \mathrm{z} 485$ to $\mathrm{m} / \mathrm{z}, 1094$ was identical to the preceding one, thus indicating the same nonreducing end. Modifications occurred at the reducing end. A fucosylated molecule gave an $[\mathrm{M}+\mathrm{H}]^{+}$ion at $\mathrm{m} / \mathrm{z}, 1461$, whereas a molecule bearing an arabinosyl substitution gave an $[\mathrm{M}+\mathrm{H}]^{+}$ion at $\mathrm{m} / \mathrm{z}$ 1447. The $[\mathrm{M}+\mathrm{H}]^{+}$ion at $\mathrm{m} / \mathrm{z} 1272$ corresponded to an unsubstituted molecule at the reducing end, which also lacked the carbamoyl group at the other end (Mergaert et al. 1993). Finally, fraction PIIf (Fig. 1E) showed an $[\mathrm{M}+\mathrm{H}]^{+}$ion at $m / z 1593$, corresponding to a molecule bearing both an arabinosyl and a fucosyl residue on the reducing end. All of these molecules were pentameric LCOs. The corresponding tetrameric analogs were found in the fractions PIIa, PIIb, and PIIc (Table 1).

To determine the carbohydrate composition of the novel compounds, PId, PIe, and PIf fractions of ORS571 were selected as representative samples. After hydrolysis, alditol acetates were prepared as described by York et al. (1986) and separated by capillary gas chromatography (GC) on an OV1 column $(0.32 \mathrm{~mm} \times 30 \mathrm{~m})$ with $\mathrm{He}$ as the carrier gas and a temperature gradient from 120 to $240^{\circ} \mathrm{C}$ at $2^{\circ} \mathrm{C} / \mathrm{min}$. Compounds were identified both by co-injection with authentic standards and by GC-MS (electron impact ionization). All three fractions contained GlcNAc and $N$-methyl- $N$-acetyl-

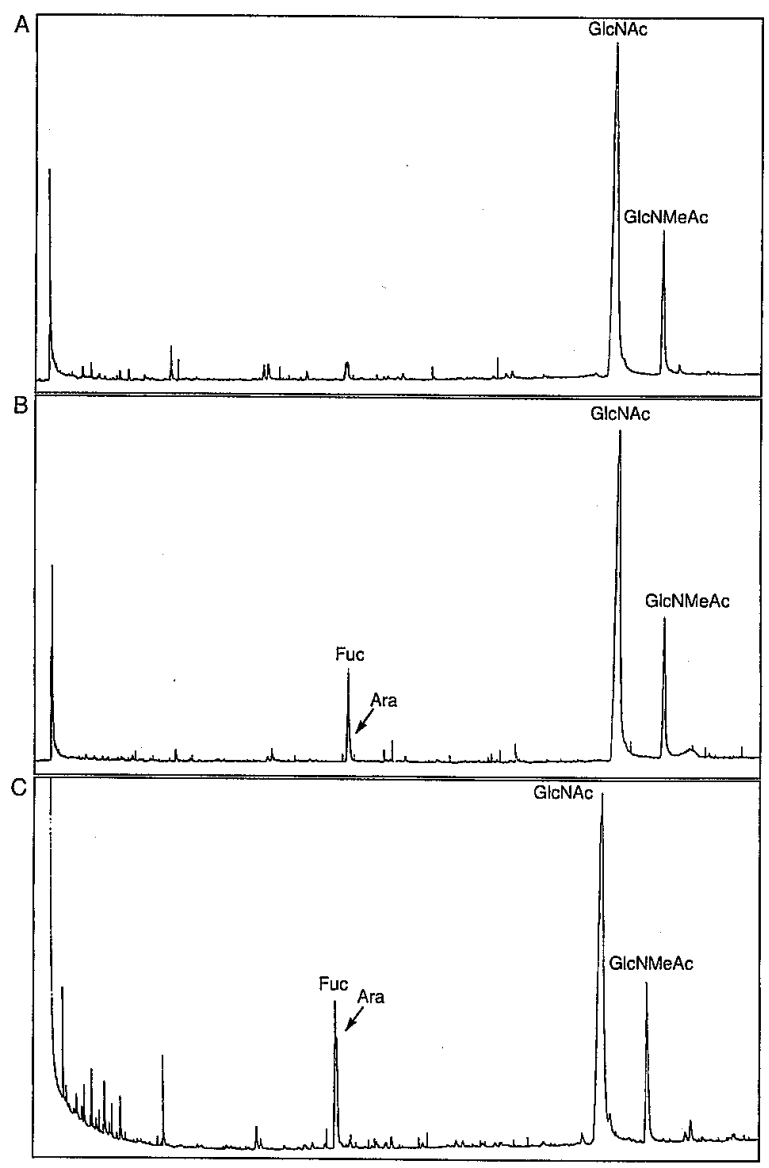

Fig. 4. Gas chromatograms of alditol acetates prepared from $\mathbf{A}, \mathrm{PId}, \mathbf{B}$, PIe, and C, PIf. GlcNAc $=N$-acetylglucosamine; GlcNMeAc $=N$ methyl- $N$-acetylglucosamine; Fuc $=$ fucose $;$ and Ara $=$ arabinose. glucosamine (GlcNMeAc). In addition, PIe contained fucose and a small amount of arabinose. PIf contained fucose and arabinose in equal amounts (Fig. 4). These results are in agreement with the FAB-MS data listed in Table 1.

The fatty acids of the RP-HPLC peaks PI and PII were analyzed as methyl esters with GC as previously described (Mergaert et al. 1995). In fraction PI, mainly vaccenic acid (C18:1) was identified, but a small amount of palmitic acid (C16:0) was also present. In fraction PII, stearic acid (C18:0) was the only fatty acid present.

The locations of the arabinosyl and fucosyl branches on the reducing GlcNAc residue were determined by methylation analysis (data not shown). The preparation of the partially methylated alditol acetates was performed as described by Ciucanu and Kerek (1984). The partially methylated alditol acetates were analyzed with GC-MS in electron impact and ammonia chemical ionization mode. In fraction PIe of ORS571 (containing mainly fucosylated LCOs) (Table 1), the glucosaminyl residue at the reducing end was found protected at O-6, indicating an O-6 fucosyl branch. In contrast, for PIe of ORS571(pRG70) containing arabinosylated LCOs only (Table 1), the reducing glucosaminyl end was found partially protected on O-6 and partially protected on O-3. Thus, the arabinosyl substitution occurred randomly at either site. In the previous experiments (Mergaert et al. 1996), we detected species with arabinose only on O-6. This discrepancy can be attributed to different culture conditions that induced a much less abundant population of arabinosylated LCOs, and O-3modified molecules could have escaped our attention.

Fraction PIf contained LCOs carrying both an arabinosyl and a fucosyl branch (Table 1). In this case, the methylation analysis demonstrated that the reducing end was branched at both O-3 and O-6. To discriminate between the possibilities of an arabinosyl branch at O-3 and a fucosyl branch at O-6 or vice versa, we treated these LCOs with an $\alpha-1,6$ fucosidase purified from bovine epididymides (Oxford GlycoSystems, Abingdon, UK). PIf ( $32 \mu \mathrm{g})$ was incubated at $37^{\circ} \mathrm{C}$ for $48 \mathrm{~h}$ in $500 \mu \mathrm{l}$ of $100 \mathrm{mM}$ sodium citrate-phosphate buffer $(\mathrm{pH} \mathrm{6})$ in the presence of 0.1 units of fucosidase. This resulted in a migration shift on the polyol HPLC column, and the product of the enzymatic digestion migrated at the position of PIe, indicating that the enzyme removed the fucosyl group of the substrate. This was confirmed by FAB-MS, showing that the substrate with an $[\mathrm{M}+\mathrm{H}]^{+}$ion at $m / z, 1591$ was converted into a product with an $[\mathrm{M}+\mathrm{H}]^{+}$ion at $\mathrm{m} / z, 1445$. During a first incubation, approximately $80 \%$ of the product was converted. When the remaining $20 \%$, repurified with polyol HPLC, was incubated a second time, again approximately $80 \%$ was converted, indicating that partial digestion resulted from formation of an equilibrium and that at least $96 \%$ of the substrate could be defucosylated by the enzyme. The specificity of the enzyme for $\alpha-1,6$-linked fucosyl residues suggested that the fucosyl branch in these molecules was located on O-6 and by default the arabinosyl branch was on O-3. A methylation analysis on the reaction products confirmed that the arabinosyl branch was indeed located on O-3 (data not shown).

In addition, the methylation studies demonstrated that the arabinosyl and fucosyl residues on NodARc were in the furanosyl and pyranosyl forms, respectively.

In conclusion, different substitution modes occur at the reducing end of NodARc. It can i) be unsubstituted or 
branched on O-6 with ii) an arabinosyl or iii) fucosyl sugar, iv) bear an arabinosyl sugar on O-3, or v) be doubly substituted with fucose on O-6 and arabinose on O-3. The relative amounts of these molecules are influenced by the bacterial growth conditions, an observation that might have implications for the Nod factors produced in the rhizosphere and in plants where bacteria could encounter different oxygen and nutrient conditions.

Recently, LCOs have been characterized for Sinorhizobium teranga and $S$. saheli species, which, like A. caulinodans, are symbionts of $S$. rostrata (J. Lorquin et al., unpublished data). These strains produce a similar collection of arabinosylated and fucosylated Nod factors. These structures may thus be important for $S$. rostrata nodulation. To determine the significance of the glycosylations for biological activity, the Nod factors of HPLC fractions PId, PIe, and PIf were assayed for their capacity to induce root hair formation at the bases of lateral roots of $S$. rostrata, which is one of the macroscopically visible effects of Nod factor application (Mergaert et al. 1993). Roots were inoculated with Nod factors at concentrations of $10^{-8}$ to $10^{-11} \mathrm{M}$ and root hairs were stained 1 week later as previously described (Mergaert et al. 1993). The response of the roots to the treatments was scored under a binocular by counting the number of lateral roots that reacted by forming new root hairs at the bases; the mean of five plants was calculated for each treatment. The experiment was conducted in triplicate, and each time a similar result was obtained. Glycosylated Nod factors (fractions PIe and PIf)

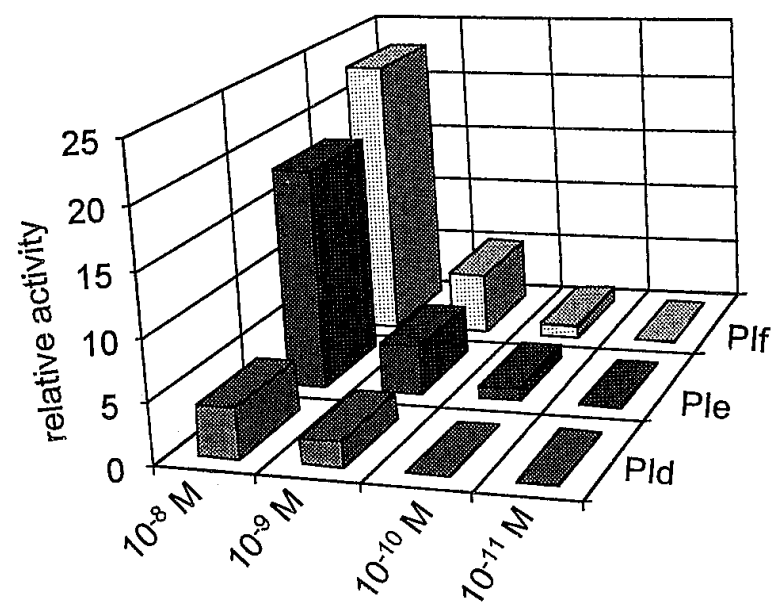

Fig. 5. Root hair formation assay on Sesbania rostrata. High-pressure liquid chromatography fractions PId (unglycosylated Nod factors), PIe (monoglycosylated Nod factors), and PIf (double glycosylated Nod factors) were assayed at the indicated concentrations on the roots of $S$. rostrata, and the root hair formation response was scored by counting the number of lateral roots with root hairs at the bases. The values correspond to the response per plant and are the means of five plants. were more active than unglycosylated Nod factors (fraction PId), but there was no great difference between Nod factors with one or two branches (Fig. 5). In this study, we did not test the PII Nod factors, but no differences in activity between the PI and PII fractions had been detected previously (Mergaert et al. 1993). These results provide a direct indication of a role for glycosylations in biological activity, which does not exclude the possibility that unglycosylated Nod factors also have a distinct role in nodulation. Use of other bioassays, determination of the sensitivity of Nod factors to plant hydrolases, and the nodulation behavior of nod gene mutants will further contribute to the elucidation of the function of the methyl, carbamoyl, arabinosyl, and fucosyl modifications in the activity of the Nod factors.

\section{ACKNOWLEDGMENTS}

The authors thank Christa Verplancke for preparing the cultures and purifying the Nod factors, Martine De Cock for help with the manuscript, and Christiane Germonprez for the figures. This work was carried out with grants from the Belgian Programme on Interuniversity Poles of Attraction (Prime Minister's Office, Science Policy Programming, no. 38), in part by the European Communities' BIOTECH Programme (as part of the Project of Technological Priority 1993 to 1996), the Human Capital and Mobility Program (CHRX-CT94-0656), and the French National Center for Scientific Research-Flemish Community Exchange Program. W. D'Haeze is indebted to the Vlaams Instituut voor de Bevordering van het Wetenschappelijk-Technologisch Onderzoek in de Industrie for a predoctoral fellowship. P. Mergaert and M. Holsters are postdoctoral fellow and research director, respectively, of the Fund for Scientific Research (Flanders).

\section{LITERATURE CITED}

Ciucanu, I., and Kerek, F. 1984. A simple and rapid method for the permethylation of carbohydrates. Carbohydr. Res. 131:209-217.

Dénarié, J., Debellé, F., and Promé, J.-C. 1996. Rhizobium lipo-chitooligosaccharide nodulation factors: Signaling molecules mediating recognition and morphogenesis. Annu. Rev. Biochem. 65:503-535.

Fernández-López, M., D’Haeze, W., Mergaert, P., Verplancke, C., Promé, J.-C., Van Montagu, M., and Holsters, M. 1996. Role of nodI and nodJ in lipo-chitooligosaccharide secretion in Azorhizobium caulinodans and Escherichia coli. Mol. Microbiol. 20:993-1000.

Mergaert, P., Van Montagu, M., Promé, J.-C., and Holsters, M. 1993. Three unusual modifications, a D-arabinosyl, an $N$-methyl, and a carbamoyl group, are present on the Nod factors of Azorhizobium caulinodans strain ORS571. Proc. Natl. Acad. Sci. USA 90:15511555 .

Mergaert, P., D’Haeze, W., Geelen, D., Promé, D., Van Montagu, M., Promé, J.-C., and Holsters, M. 1995. Biosynthesis of Azorhizobium caulinodans Nod factors. Study of the activity of the NodABCS proteins by expression of the genes in Escherichia coli. J. Biol. Chem. 270:29217-29223.

Mergaert, P., D’Haeze, W., Fernández-López, M., Geelen, D., Goethals, K., Promé, J.-C., Van Montagu, M., and Holsters, M. 1996. Fucosylation and arabinosylation of Nod factors in Azorhizobium caulinodans: Involvement of nolK, nodZ, and noeC, and/or downstream genes. Mol. Microbiol. 21:409-419.

York, W. S., Darvill, A. G., McNeil, M., Stevenson, T. T., and Albersheim, P. 1986. Isolation and characterization of plant cell walls and cell wall components. Methods Enzymol. 118:3-40. 